\title{
Mathematical Modeling of Polymer Loading Process in Extruders
}

\author{
K. Zelensky, Ev. Nastenko, V. Bolhovitin, O. Pavlov
}

\begin{abstract}
Based on the state of the problem related to highquality and reliable insulation coating of high-voltage cables, the problems of mathematical modeling of pro-cesses in the loading zone and the delay of the polymer mixture in a single-screw extruder are formulated, which is crucial in terms of providing a high-quality insulation coating. An iterative numerical-analytical method for solving the corresponding nonlinear boundary value problems is proposed, based on the use of finite integral transformations in the spatial and temporal domains and their implementation.
\end{abstract}

Keywords: Bessel functions, extruder, integral transformations, mathematical model, numerical-analytical method, Navier-Stokes equation, polymer, screw.

\section{I.INTRODUCTION}

Due to the high productivity of the extruders, their substantial value, and the high cost of polymeric materials, experimental studies on equipment upgrades and technological regimes lead to costly materials and time costs. This leads to the development of theoretical foundations of the processes under study. One of the main tools that helps to get the results you need and allows you to minimize expensive field tests is mathematical modeling. Although the vast majority of theoretical work on the description of processes of heat transfer is based on the classical Navier - Stokes equations [1,2,3], at the same time, existing approaches to the modification of the processes of flow, heat exchange and phase transformation of polymers in channels of extrusion equipment [4] do not provide a qualitative and quantitative analysis of the processes, since the vast majority of them are formulated either in onedimensional formulation [5-11] or formulated in threedimensional formulation, but the corresponding boundaryvalue problems are not given or solved communications link to know " standard " packages numerical solution, which by their nature are not designed to solve nonlinear models. In addition, in our view, when setting these problems, the boundary conditions inherent in such a complex physical phenomenon are incorrectly formulated [4]. In addition, a number of problems have not been solved in the theory of plastic extrusion.

Revised Manuscript Received on February 05, 2020.

* Correspondence Author

K. Zelensky*, Associate Professor, Department of Biomedical Cybernetics, National Polytechnic University, Ukraine.

Evgen Nastenko, Senior Researcher, Department of Biomedical Cybernetics, National Polytechnic University, Ukraine.

V. Bolhovitin, Lecturer, Department of Computer Systems and Networks, Institute of Computer Technology of the University, Ukraine

A. Pavlov., Senior Lecturer, Department of Computer Science, National Technical University, Ukraine.

(C) The Authors. Published by Blue Eyes Intelligence Engineering and Sciences Publication (BEIESP). This is an open access article under the CC BY-NC-ND license (http://creativecommons.org/licenses/by-nc-nd/4.0/)
As a rule, the rheological states of the polymer mixtures are not taken into account, the effect of the heat transfer processes in the auger on the processes of plastic extrusion are practically not considered.

Addressing these issues is important in terms of improving product quality, improving work efficiency when designing and upgrading extrusion equipment, and improving technological modes.

\section{II.METHODOLOGY AND MATERIALS}

\section{II.1 Object description}

In Fig. 1 [5], provides a diagram of an extruder for coating plastic wires

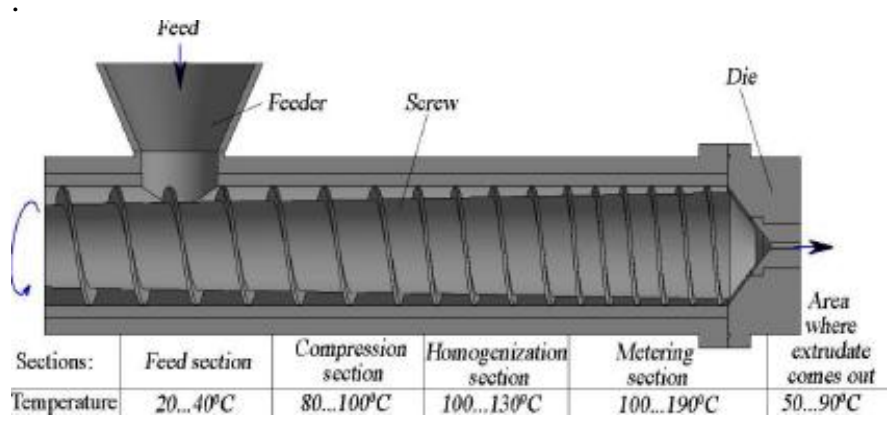

Fig 1. Schematic of single screw extruder

The object of this study is the loading zone and the delay zone in which the polymer mixture is heated to a temperature close to the melting point of the polymer.

The main source of heating of the body of the extruder is induction heating. The density of internal heat sources is the electromagnetic energy released per unit time per unit volume. Due to the surface effect, the distribution of internal heat sources is significantly heterogeneous and depends on the electrophysical properties of the load, which change during heating.

The whole process of heating is divided into intervals, in each of which the loading properties are assumed unchanged.

$$
L_{i}(m)=\{0.9 ; 1.45 ; 2.05 ; 2.65\} .
$$

\section{II.2 Heating the extruder housing}

The distribution of the temperature field of the body $T_{k}$ is described by the equation of thermal conductivity, which in the cylindrical coordinate system has the form

$$
\frac{\partial T_{k}}{\partial t}=a_{k}\left(\frac{1}{r} \frac{\partial}{\partial r}\left(r \frac{\partial T_{k}}{\partial r}\right)+\frac{\partial^{2} T_{k}}{\partial z^{2}}\right),
$$

where $a_{k}=\lambda_{k} /\left(c_{k} \rho_{k}\right.$ is the coefficient of thermal conductivity; $\lambda_{k}, c_{k}, \rho_{k}$ is the coefficient of thermal conductivity (W/ $\left(\mathrm{m}^{\circ} \mathrm{C}\right)$,

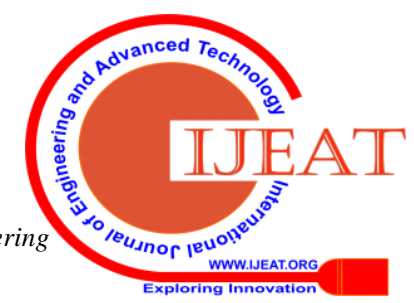




\section{Mathematical Modeling of Polymer Loading Process in Extruders}

the specific heat and density of the body material, respectively ; $g(r, z)$ is the function of the density distribution of the internal energy sources in the material, $\mathrm{W}$ / $\mathrm{m}^{3}$. Given that the depth of penetration of electromagnetic energy from the inductor is small, $\Delta \ll 1$, we assume that it acts on the outer surface of the housing $r=r_{4}$. Then the boundary conditions for the housing:

$$
\begin{aligned}
& \left.T_{k}\right|_{r=0}=T_{0 k} ;\left.\frac{\partial T_{k}}{\partial r}\right|_{r=r_{3}}=-\frac{q_{k}}{h_{1}} ; \\
& {\left.\left[\frac{\partial T_{k}}{\partial r}+h_{1}\left(T_{k}-T_{0 k}\right)\right]\right|_{r=r_{4}}=g\left(r_{4}, z\right),} \\
& {\left.\left[\frac{\partial T_{k}}{\partial z}-h_{1} T_{k}\right]\right|_{z=0}=T_{0},\left.\left[\frac{\partial T_{k}}{\partial z}+h_{1} T_{k}\right]\right|_{z=L}=0 .}
\end{aligned}
$$

where $h_{1}=\alpha_{k} / \lambda_{k} ; \alpha_{k}$ is the heat transfer coefficient of the enclosure into the environment. For the contact surface of a steel pipe with air $\alpha_{k}=9 \mathrm{~W} /\left(\mathrm{m}^{2}{ }^{\circ} \mathrm{C}\right)$.

Substitution $T=T_{k}-T_{0 k}$ reduces the problem to the equation

$$
\frac{\partial T}{\partial \tau}=a_{k}\left(\frac{1}{r} \frac{\partial}{\partial r}\left(r \frac{\partial T}{\partial r}\right)+\frac{\partial^{2} T}{\partial z^{2}}\right)
$$

with boundary conditions

$$
\begin{aligned}
& \left.T\right|_{r=0}=0 ;\left.\frac{\partial T}{\partial r}\right|_{r=r_{3}}=-\frac{q_{k}}{\lambda_{k}} ; \\
& {\left.\left[\frac{\partial T}{\partial r}+h_{1} T\right]\right|_{r=r_{4}}=g\left(r_{4}, z\right),} \\
& {\left.\left[\frac{\partial T}{\partial z}-h_{1} T\right]\right|_{z=0}=\left(1-h_{1}\right) T_{0 k},} \\
& {\left.\left[\frac{\partial T}{\partial z}+h_{1} T\right]\right|_{z=L}=h_{1} T_{0 k} .}
\end{aligned}
$$

The values of thermophysical parameters for the housing (steel) at temperature $T=300 K$ are equal to: $\rho=7845$ $\mathrm{kg} / \mathrm{m}^{3}, \quad c_{V}=0,461 \quad \mathrm{~kW} /(\mathrm{kg} \cdot C \cdot K), \lambda=58$, $\alpha=10.51 / K$. Find the temperature field of the body of the extruder on the first section of heating (the area of polymer loading).

$Z$ native conversion functions:

$$
\begin{gathered}
Z\left(\delta_{k} z\right)=\frac{1}{\|\left. Z_{k}\right|^{2}}\left[\sin \delta_{k} z-\frac{\delta_{k}}{h_{1}} \cos \delta_{k} z\right] \\
\frac{\partial \bar{T}}{\partial \tau}=\frac{1}{r} \frac{\partial}{\partial r}\left(r \frac{\partial \bar{T}}{\partial r}\right)+Q z_{k}\left(\delta_{k}\right) \\
Q z_{k}=\left[h_{1}\left(\sin \delta_{k} L-\frac{\delta_{k}}{h_{1}} \cos \delta_{k} L\right)+\left(1-h_{1}\right) \frac{\delta_{k}}{h_{1}}\right] T_{0 k}
\end{gathered}
$$

Applying to (5) the integral transformation on the variable $r$ leads to the search for the solution of the Bessel equation

$$
\frac{1}{r} \frac{\partial}{\partial r}\left(r \frac{\partial R_{n}(r)}{\partial r}\right)+\delta_{k}^{2} R_{n}(r)=0
$$

with homogeneous boundary conditions

$$
\left.\frac{d R}{d r}\right|_{r=r_{3}}=0 ;\left.\left[\frac{d R}{d r}+h_{1} R\right]\right|_{r=r_{4}}=0 .
$$

The $r$ eigenfunctions take the form:

$$
R_{n}(\beta r)=\frac{1}{\left\|R_{n}\left(\beta_{n} r\right)\right\|^{2}}\left[I_{0}(\beta r)-D_{n}\left(\beta r_{3}\right) J_{0}(\beta r)\right] \text {. }
$$

Given (6) and (4), we have an expression for the temperature field of the auger housing.

$$
\begin{aligned}
& T_{k}(r, z, t)=\sum_{n=1}^{M} \sum_{k=1}^{M} \frac{1}{\left|R_{n}\right|^{2}}\left[I_{0}\left(\beta_{n} r\right)-D_{n} J_{0}\left(\beta_{n} r\right)\right] \\
& \times Z_{k}\left(\delta_{k} z\right) \frac{G_{n k}}{\eta_{n k}^{2}}\left(1-e^{-\eta_{n k}^{2} t}\right) . \\
& \text { where } \eta_{n k}=\lambda_{k} /\left(c_{v} \rho\right)\left(\beta_{n}^{2}+\delta_{k}^{2}\right) .
\end{aligned}
$$

In Fig. 2 results of simulation of auger housing temperature field.

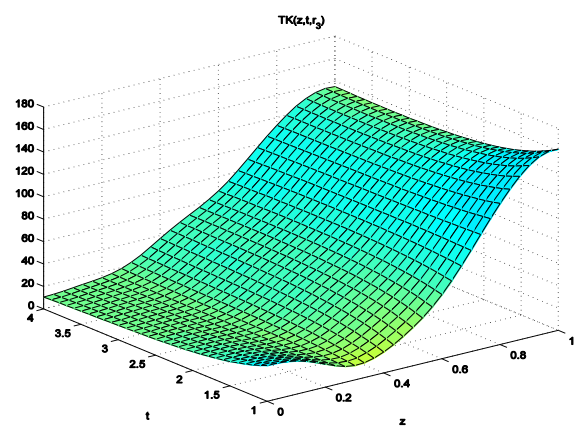

Fig.2 Screw housing temperature field at fixed $t$

\section{II.3 Simulation of Polymer Temperature Field in Loading Area}

When formulating the boundary value of the process of heating the dry polyethylene mixture at the loading area, it is necessary to take into account the presence of a rotational motion of the screw at a constant speed $V_{s n}$ with cutting at an angle $\varphi$. The heat transfer equation looks like:

$$
\begin{array}{r}
\rho_{\mathrm{p}} c_{v_{\mathrm{p}}}\left(V_{\mathrm{sn}}^{r} \frac{\partial T}{\partial r}+V_{\mathrm{sn}}^{z} \frac{\partial T}{\partial \mathrm{z}}+\frac{\partial T_{\mathrm{p}}}{\partial t}\right) \\
=\lambda_{\mathrm{p}}\left(\frac{1}{r} \frac{\partial}{\partial r}\left(r \frac{\partial T_{\mathrm{p}}}{\partial r}\right)+\frac{\partial^{2} T_{\mathrm{p}}}{\partial \mathrm{z}^{2}}\right) .
\end{array}
$$

For polyethylene, $c_{v_{\mathrm{p}}}$ is essentially dependent on the heating temperature [4]. The heat capacity coefficient is approximated by the dependence

$$
c_{v_{\mathrm{p}}}=c_{0}+c_{1} T+c_{2} T^{2} \approx 2,5-0,024 \cdot T+5,7 \cdot 10^{-4} \cdot T^{2} \text {. }
$$

At the boundary of the housing and the auger, the condition of equality of heat flows must be fulfilled

$$
\left.\lambda_{k} \frac{\partial T_{k}}{\partial r}\right|_{r=r_{3}}=\left.\lambda_{s n} \frac{\partial T_{s n}}{\partial r}\right|_{r=r_{3}} .
$$

Given (7) we have a boundary condition at $r=r_{3}$ for the screw temperature:

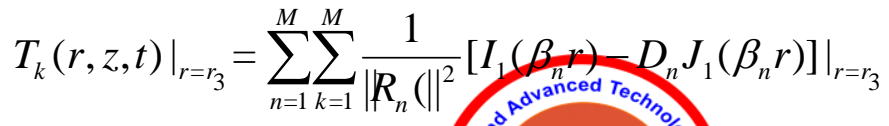




$$
\begin{gathered}
\times Z_{k}\left(\delta_{k} z\right) \frac{G_{n k}}{\left(\eta_{n k}^{2}\right)}\left(1-e^{-\eta_{n k}^{2} t}\right) . \\
\left.T_{\mathrm{sn}}\right|_{z=0}=T_{s n}^{0}, \\
{\left.\left[\frac{\partial T_{\mathrm{sn}}}{\partial z}+h_{2} T_{\mathrm{sn}}\right]\right|_{z=L_{1}}=0 .}
\end{gathered}
$$

The temperature field of the polymer mixture in the loading zone is described by the boundary value problem (8) - (13). Given the dependence of the coefficient of thermal capacity of the polymer on the temperature $C_{v p}$, the thermal conductivity equation for the polymer in the loading zone can be written as:

$$
\frac{\partial T_{\mathrm{p}}}{\partial t}=a_{\mathrm{p}}\left(\frac{1}{r} \frac{\partial}{\partial r}\left(r \frac{\partial T_{\mathrm{p}}}{\partial r}\right)+\frac{\partial^{2} T_{\mathrm{p}}}{\partial \mathrm{z}^{2}}\right)+N T_{\mathrm{p}}(r, z, t),
$$

where $a_{\mathrm{p}}=\lambda_{\mathrm{p}} /\left(\rho c_{01}\right)$.

$$
N T_{\mathrm{p}}(r, z, t)=\left[c_{11}^{\prime} T_{\mathrm{p}}+c_{21}^{\prime} T_{\mathrm{p}}^{2}\right] \frac{\partial T_{\mathrm{p}}}{\partial t},
$$

where $c_{11}^{\prime}=-c_{11} / c_{01}, c_{21}^{\prime}=-c_{21} / c_{01}$.

Since the equation (1) is nonlinear, we will look for an iteration scheme. Solution of the linear part of the equation

$$
\frac{\partial T_{\mathrm{p}}^{(0)}}{\partial t}=a_{\mathrm{i} 0}\left[\frac{1}{r} \frac{\partial}{\partial r}\left(r \frac{\partial T_{\mathrm{p}}^{(0)}}{\partial r}\right)+\frac{\partial^{2} T_{\mathrm{p}}^{(0)}}{\partial \mathrm{z}^{2}}\right]
$$

The difference is the condition at the boundary $r=r_{3}$, which gives the expression for the temperature field at that boundary (11). When applying integral transforms for spatial variables to a boundary-value problem (14) - (16), we take into account (11) the following condition:

$$
\begin{gathered}
\overline{T^{k}}\left(r_{3}, \gamma_{k}, t\right)==\sum_{n=1}^{M}\left[I_{0}\left(\beta_{n} r_{3}\right)-D_{n}\left(r_{2}\right) J_{0}\left(\beta_{n} r_{3}\right)\right] \\
\times\left[\sum_{n_{1}=1}^{M} \sum_{k=1}^{M} \sum_{k_{1}=1}^{M} U G_{n_{1}}, k, k_{1}\left(1-e^{-\eta_{n_{1}}^{2} k_{1} t}\right)\right] .
\end{gathered}
$$

Using this expression as a boundary condition for solving the problem of heat transfer (14), (12), (13) and (17) results in operations with $M \times N \times N$ components of appearance $U G_{n_{1}}, k, k_{1}\left(1-e^{-\eta_{n_{1}}^{2} k_{1}^{t}}\right)$, making it virtually impossible to use them again. So let's simplify this expression.

$$
\begin{aligned}
& \overline{T^{k}}\left(r_{3}, \gamma_{k}, t\right) \approx \sum_{n_{1}=1}^{M} \sum_{k=1}^{M} \sum_{k_{1}=1}^{M} U G_{n_{1}, k, k_{1}}\left(1-e^{-\eta_{n_{1}}^{2} k_{1} t}\right) \\
& =\sum_{n=1}^{N} \sum_{k=1}^{M}\left[g r_{0}^{n, k}\right. \\
& +e^{-\alpha^{n, k} t}\left(g r_{1}^{n, k} \sin \left(\omega^{n, k} t\right)+g r_{2}^{n, k} \cos \left(\omega^{n, k} t\right)\right] .
\end{aligned}
$$

The algorithm behind this simplification is outlined in the section. The error that naturally arises from such simplification is offset by an iterative process when solving a nonlinear problem. Write the heat transfer equation in the screw (the temperature field of the polymer mixture) after applying the transformations on the spatial variables $z, r$ :

$$
\overline{\overline{T 0}}_{n, k}(t)+\eta_{n, k} \overline{\bar{T}}_{n, k}(t)=g z_{n, k}+G r_{n, k}(t) .
$$

We will have $t$ in image space

$$
\begin{aligned}
& \overline{\overline{T 0}}_{n, k}(p)=\frac{1}{p+\eta_{n, k}}\left[\frac{g z_{n, k}}{p}\right. \\
& \left.+\frac{\left(g r_{0}^{n, k} \omega^{n, k}-g r_{1}^{n, k} \alpha^{n, k}\right)+g r_{1}^{n, k}\left(p+\alpha^{n, k}\right)}{\left(p+\alpha^{n, k}\right)^{2}+\left(\omega^{n, k}\right)^{2}}\right] \\
& =\frac{g z_{n, k}}{\eta_{n, k}} \frac{1}{p}+\frac{t 0_{0}^{n, k}+t 0_{1}^{n, k} p}{t 0_{3}^{n, k}+t 0_{4}^{n, k} p+p^{2}} .
\end{aligned}
$$

In the space of originals we have

$$
\begin{array}{r}
T^{(0)}(r, z, t)=\sum_{n=1}^{N} \sum_{k=1}^{M} R_{n}(r) Z_{k}(z) T 0(t) . \\
T 0(t)=g z 1^{n, k} \\
+e^{-\alpha^{n, k} t}\left[t 1_{0}^{n, k} \sin \left(\omega^{n, k} t\right)+t 1_{1}^{n, k} \cos \left(\omega^{n, k} t\right)\right]
\end{array}
$$

This expression takes into account approximate equality

$$
\begin{gathered}
\frac{1}{p+\eta^{n, k}} \frac{t 0_{0}^{n, k}+t 0_{1}^{n, k} p}{t 0_{3}^{n, k}+t 0_{4}^{n, k} p+p^{2}} \\
\rightleftharpoons e^{-\alpha^{n, k} t}\left[t 1_{0}^{n, k} \sin \left(\omega^{n, k} t\right)+t 1_{1}^{n, k} \cos \left(\omega^{n, k} t\right)\right]
\end{gathered}
$$

After obtaining the solution of the equation in the form (21), taking into account (22), we will look for the solution of the equation (14) according to the iterative scheme. Recall that

$$
N T_{\mathrm{p}}^{(1)}(r, z, t)=\left[c_{11}^{\prime} T_{\mathrm{p}}^{(0)}+c_{21}^{\prime}\left(T T^{(0)}\right)_{\mathrm{p}}^{2}\right] \frac{\partial T_{\mathrm{p}}}{\partial t},
$$

The general scheme for solving the nonlinear equation is written in the form

$T_{\mathrm{p}}^{(m)}(r, z, t)=T_{\mathrm{p}}^{(0)}(r, z, t)+N T_{\mathrm{p}}^{(m-1)}(r, z, t), m=1,2, \mathrm{~K}$

We replace the expression (20) with (15).

$T_{\mathrm{p}}^{(0)} \frac{\partial T_{\mathrm{p}}^{(0)}}{\partial t}=\sum_{n_{1}=1_{k_{1}}=1}^{M} \sum_{n_{1}}^{M}\left(\beta_{n_{1}}^{p} r\right) Z_{k_{1}}\left(\left(\delta^{p}\right)_{k_{1}} z\right)$

$\times \frac{G_{n_{1} k_{1}}^{p}}{\left(\eta^{p}\right)_{n_{1}}^{2} k_{1}}\left(1-e^{-\left(\eta^{p}\right)_{n_{1}}^{2} k_{1} t}\right)$

$\times \sum_{n_{2}}^{M} \sum_{k_{2}=1}^{M} R_{n_{2}}^{p}\left(\beta_{n_{2}}^{p} r\right) Z_{k_{2}}\left(\delta_{k_{2}}^{p} z\right)$

$\times \frac{G_{n_{2} k_{2}}^{p}}{\left(\eta^{p}\right)_{n_{2} k_{2}}^{2}}\left(\left(\eta^{p}\right)_{n_{2} k_{2}}^{2} e^{-\left(\eta^{p}\right)_{n_{2}}^{2} k_{2} t}\right)$;

$\eta_{n k}^{p}=a_{k}\left[\left(\beta^{p}\right)_{n}^{2}+\left(\delta^{p}\right)_{k}^{2}\right]=a_{k} \gamma_{n k}^{2}, a_{k}=\lambda^{p} /\left(\rho^{p} c_{01}\right)$.

$\left(T_{\mathrm{p}}^{(0)}\right)^{2} \frac{\partial T_{\mathrm{p}}^{(0)}}{\partial t}=\sum_{n_{1}=1}^{M} \sum_{k_{1}=1}^{M} R_{n_{1}}^{p}\left(\beta_{n_{1}}^{p} r\right) Z_{k_{1}}\left(\left(\delta^{p}\right)_{k_{1}} z\right)$

Published By: 


$$
\begin{gathered}
\times \frac{G_{n_{1} k_{1}}^{p}}{\left(\eta^{p}\right)_{n_{1} k_{1}}^{2}}\left(1-e^{-\left(\eta^{p}\right)_{n_{1}}^{2} k_{1} t}\right) \\
\times \sum_{n_{2}=1}^{M} \sum_{k_{2}=1}^{M} R_{n_{2}}^{p}\left(\beta_{n_{2}}^{p} r\right) Z_{k_{2}}\left(\left(\delta^{p}\right)_{k_{2}} z\right) \\
\left.\times \frac{G_{n_{2} k_{2}}^{p}}{\left(\eta^{p}\right)_{n_{2} k_{2}}^{2}}\left(1-e^{-\left(\eta^{p}\right)_{n_{2} k_{2}}^{t}}\right) \times \sum_{n_{3}=k_{k_{3}}=1}^{M} \sum_{n_{2}}^{M} R_{n_{3}}^{p} r\right) Z_{k_{3}}\left(\delta_{k_{3}}^{p} z\right) \\
\times \frac{G_{n_{3} k_{3}}^{p}}{\left(\eta^{p}\right)_{n_{3} k_{3}}^{2}}\left(\left(\eta^{p}\right)_{n_{3} k_{3}}^{2} e^{-\left(\eta^{p}\right)_{n_{3} k_{3}}^{2}}\right) .
\end{gathered}
$$

Applying integral transforms to (14) on spatial variables leads to the following equation:

$$
{\overline{\overline{T^{(1)}}}}_{n k}(p)={\overline{\overline{\overline{T^{(0)}}}}}_{n k}(p)+{\overline{\overline{\overline{N T^{(0)}}}}}_{\mathrm{p}}(r, z, t) .
$$

Further, given the temperature field of the polymer mixture, the index $p$ is omitted to simplify the calculations. Integral transformations on the variables $Z$ and $r$ (after replacing (26), (27) in (15)) lead to the calculation of integrals from the products eigenfunctions on the variables $Z$ and $r$. Unlike the integration of the product of $Z_{k_{i}}\left(\delta_{k_{i}} z\right)$, which is not difficult, to integrate the product of $R_{n_{i}}\left(\beta_{n_{j}} r\right)$

Bessel functions of the second kind, these functions are approximated the sum of second-degree fractional-rational functions, after which the process of integration is reduced to the integration of second-order fractional-rational expressions. An algorithm for such transformations is described in [15,17]. Therefore, after applying the integral transformations for spatial variables to the nonlinear part of the equation (28), we obtain:

$$
\begin{aligned}
& {\overline{\overline{\overline{T^{(1)}}}}}_{n k}(p)={\overline{\overline{\overline{T^{(0)}}}}}_{n k}(p) \\
& +\mathcal{L}_{t}\left\{c_{11} \overline{\bar{G}}_{n_{1} k_{1}} \overline{\bar{G}}_{n_{2} k_{2}}\left(1-e^{-\eta_{n_{1}}^{2} k_{1} t}\right) \eta_{n_{2} k_{2}}^{2} e^{-\eta_{n_{2}}^{2} k_{2}^{t}}\right. \\
& +c_{21} n r_{n, n_{1}} n_{2} n_{3} n z_{k, k_{1}} k_{2} k_{3} G_{n_{1} k_{1}} G_{n_{2} k_{2}} G_{n_{3}} k_{3} \\
& \left.\times\left(1-e^{-\eta_{n_{1}}^{2} k_{1} t}\right)\left(1-e^{-\eta_{n_{2}}^{2} k_{2} t}\right) \eta_{n_{3} k_{3}^{2}}^{2} e^{-\eta_{n_{3}}^{2} k_{3} t}\right\} \text {. } \\
& {\overline{\overline{\overline{T^{(1)}}}}}_{n k}(p)={\overline{\overline{\overline{T^{(0)}}}}}_{n k}(p) \\
& +\mathcal{L}_{t}\left\{\sum_{n_{1}, n_{2}=1_{k_{1}}, k_{2}=1}^{M}\left[d^{1}\left(e^{-s^{1} t}-e^{-s^{2} t}\right)\right]\right. \\
& +\sum_{n_{1}, n_{2}=1}^{N} \sum_{n_{3}=1_{k_{1}}, k_{2}=1_{k_{3}}=1}^{M} \sum^{M}\left[d^{2}\left(e^{-s^{3} t}-e^{-s^{4} t}+e^{-s^{5} t}\right)\right]
\end{aligned}
$$

Recall that due to the notation introduced, the coefficients $d_{1}, d_{2}, s_{i}, i=\overline{1,5}$, depend on the eigenvalues of the spatial variables. Apply to the Laplace transform equation over time (the initial condition is zero). We have:

$$
\begin{aligned}
& {\overline{\overline{T^{(1)}}}}_{n k}(p)={\overline{\overline{\overline{T^{(0)}}}}}_{n k}(p)+\frac{1}{p+\eta_{n k}}\left[d^{1}\left(\frac{1}{p+s^{1}}-\frac{1}{p+s^{2}}\right)\right. \\
& \left.+d^{2}\left(\frac{1}{p+s^{3}}-\frac{1}{p+s^{4}}+\frac{1}{p+s^{5}}\right)\right]
\end{aligned}
$$

The expression in square brackets ((20)) omits (for clarity) the sums of the indices $n_{1}, n_{2}, n_{3}$ and $k_{1}, k_{2}, k_{3}$. For one " point", the inverse Laplace transform to ((20)) gives the expression:

$$
{\overline{\overline{T^{(1)}}}}_{n k}(t)={\overline{\overline{T^{(0)}}}}_{n k}(t)+E_{0} e^{-\eta n k} t+\sum_{i=1}^{5} E_{i} e^{-s i t},
$$

Summation by the indices of the right-hand side of the equation (31) will result in such an expression with respect to the operator $p$, which makes it impossible for its practical implementation by means of computer engineering. For the solution in the second approximation we have to substitute in (29) which will result in the production of expression expressions (31), etc., that is, the increase of constituents relative to elementary chains in geometric progression.

This leads to the development of algorithms that prevent this growth. The accumulated errors can be compensated by additional iterations in the corresponding algorithm.

So, let's go back to the equation (31) and consider the expression in square brackets, taking into account thsummation of the index $k_{2}$ $\sum_{k_{2}}^{M} d_{k_{2}}^{1}\left(\frac{1}{p+s_{k_{2}}^{1}}-\frac{1}{p+s_{k_{2}}^{2}}\right)+\sum_{k_{2}}^{M M} \sum_{k_{3}}^{M} d_{k_{2}}^{2} k_{3}\left(\frac{1}{p+s_{k_{2} k_{3}}^{3}}-\frac{1}{p+s_{k_{2} k_{3}}^{4}}+\frac{1}{p+s_{k_{2} k_{3}}^{5}}\right)$
$\approx \frac{b_{3}^{k_{1}}+b_{4}^{k_{1}} p}{b_{0}^{k_{1}}+b_{1}^{k_{1}} p+b_{2}^{k_{1}} p^{2}}$.

In turn, the resulting expression can be approximated by the same pattern

$$
\sum_{k_{1}=1}^{M} \frac{b_{3}^{k_{1}}+b_{4}^{k_{1}} p}{b_{0}^{k_{1}}+b_{1}^{k_{1}} p+b_{2}^{k_{1}} p^{2}} \approx \frac{c_{3}^{n_{2}}+c_{4}^{n_{2}} p}{c_{0}^{n_{2}}+c_{1}^{n_{2}} p+c_{2}^{n_{2}} p^{2}} .
$$

Given that the eigenvalues of $\eta^{2}$ increase quite rapidly with increasing $N$ and $M$, we can restrict ourselves to $N=M=4$. An algorithm for such simplified fractional expressions is given in the section that results in:

$$
D_{n k}^{(0)}(p)=\sum_{n k}^{M} \frac{d_{3}^{n k}+d_{4}^{n k} p}{d_{0}^{n k}+d_{1}^{n k} p+d_{2}^{n k} p^{2}} .
$$

This expression corresponds to the original (in the space of integral transformations by spatial variables)

$$
D_{n k}^{(0)}(t)=\sum_{n=1, k=1}^{M} e^{-\alpha_{n k} t}\left(e 1_{n k} f_{1} \omega_{n k} t+e 2_{n k} f_{2} \omega_{n k} t,\right) .
$$

Then the solution in the first approximation will take the form

$$
T^{(1)}(r, z, t)=\sum_{n=1}^{M} \sum_{k=1}^{M} R\left(\delta_{n} r\right) Z\left(\beta_{k} z\right)
$$

Published By: 


$$
\times\left[\frac{G_{n k}}{\eta_{n k}}\left(1-e^{-\eta_{n k} t}\right)+D_{n k}^{(0)}(t)\right] .
$$

Further approximations are implemented in the same way:

$$
\begin{aligned}
& T^{(m)}(r, z, t)=\sum_{n=1}^{M} \sum_{k=1}^{M} R\left(\delta_{n} r\right) Z\left(\beta_{k} z\right) \\
& \times\left[\frac{G_{n k}}{\eta_{n k}}\left(1-e^{-\eta_{n k} t}\right)+D_{n k}^{(m-1)}(t)\right] .
\end{aligned}
$$

In Fig. 3,4 graphs of the temperature field of the polymer in the loading zone at the first and third iteration are shown. Third approximation error is $0.05 \%$

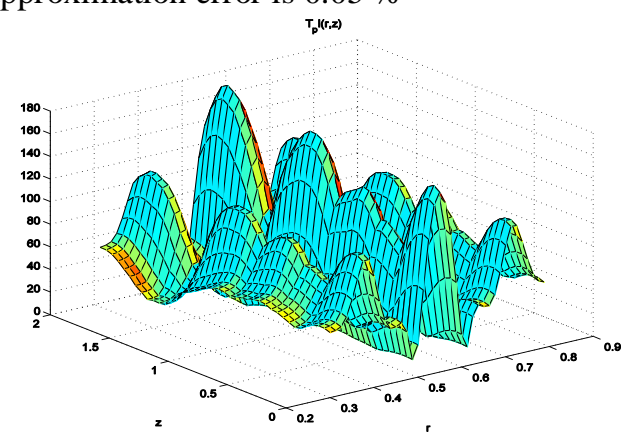

Fig 3 Screw housing temperature field at fixed $t$ - first iteration

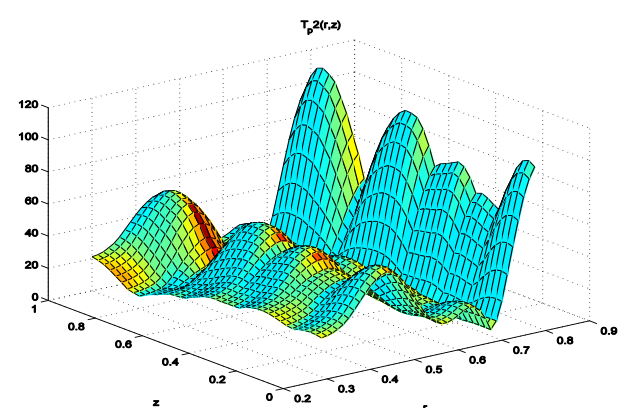

Fig.4 Screw housing temperature for fixed $t$ - third iteration

\section{II.4 Delayed Processes}

After heating the polyethylene mixture in the loading zone to the melting point in the delay zone, a boundary layer of polymer melt is formed. In this zone, the mixture is transitioned from solid to liquid in the boundary region to form a thin film of molten polymer.

This problem is mathematically described by two-phase equations of thermal conductivity in the solid and liquid phases, known as the two-phase Stefan type problem.

The peculiarity of this problem is the abrupt change in the coefficient of heat capacity at the boundary of the transition of the mixture from solid to liquid.

Many problems are devoted to solving problems of the Stefan type. They are all based on the use of difference schemes in mathematical physics and are usually limited to solving one-dimensional relative spatial variables by chance. Consider the problem of transitioning a mixture from a solid state to a liquid with the formation of a thin melt film in the boundary zone of the screw - housing.

From the point of view of computational algorithms, the fact that Stefan's problem permits the formulation under which conditions at the phase transition boundary are included in the thermal conductivity equation is important [17]. This

formulation is called enthalpy.

The domain $\Omega^{+}(t)$ of the liquid phase, where the temperature exceeds the phase transition temperature $T^{*}$, is $\Omega^{+}(t)=\left\{(x, y, z)\right.$ in $\left.\Omega, T(x, y, z)>T^{*}\right\}$. Accordingly, the domain $\Omega^{-}(t)=\left\{(x, y, z) \in \Omega, T(x, y, z)<T^{*}\right\}$. We use the same notation for thermophysical values in each phase.

In the solid phase, we have the equation of thermal conductivity

$c^{-} \rho^{-} \frac{\partial T^{-}}{\partial t}=\operatorname{div}\left(k^{-} \operatorname{grad} T^{-}\right)+f^{-},(x, y, z) \in Q^{-}$,

where $Q^{-}=\left\{(x, y, z, t) \mid(x, y, z) \in \Omega^{-}, 0<t<t_{\max }\right\}$.

Given the convective transfer in the liquid phase,

$$
\begin{aligned}
& c^{+} \rho^{+}\left(\frac{\partial T^{+}}{\partial t}+\operatorname{vgrad} T^{+}\right) \\
& =\operatorname{div}\left(k^{+} \operatorname{grad} T^{+}\right)+f^{+}, \quad(x, y, z) \in Q^{+} . \\
& {[T]=0, \quad(x, y, z) \in S .}
\end{aligned}
$$

where [.] denotes a jump in the boundary of the $S$ phase transition.

The phase transition is accompanied by the release / absorption of a certain amount of heat. Therefore, the thermal flux at the boundary of the phase transition is discontinuous and is determined by the magnitude

$$
\left[\frac{\partial T}{\partial n}\right]=-L V_{n},(x, y, z) \in S .
$$

Here $L$ is the enthalpy of the phase transition, $V_{n}$ the velocity of the phase transition boundary at normal.

At the boundary of the phase transition conditions of the first kind are fulfilled:

$$
T(x, y, z, t)=T^{*},(x, y, z) \in S(t) .
$$

Conditions (37)-(39) -- Stefan conditions, and the corresponding problem for the equations (35), (36) is called the Stefan problem. From the point of view of constructing efficient algorithms, it is important that the Stefan problem admits a generalized formulation under which the conditions (37)-(39) are included in the thermal conductivity equation itself.

$c \rho\left(\frac{\partial T}{\partial t}+\operatorname{vgrad} T\right)=\operatorname{div}(k \operatorname{grad} T)+f, \quad(x, y, z) \in Q$.

Near the boundary of the phase transition, we introduce a local orthogonal coordinate system $\left(x^{\prime}, y^{\prime}, z^{\prime}\right)$, whose metric coefficients are equal to one. In these new coordinates, the surface $\delta$-function $\delta_{S}$ is $\delta_{S}=\delta\left(x^{\prime}-x_{0}^{\prime}\right)$, where the equation $x^{\prime}=x_{0}^{\prime}$ defines the limit $S$. Similarly, for the speed of free boundary motion we have $V_{n}=d x^{\prime} / d t$. The Stefan condition ( ref stef.05) corresponds to the fact that in the new coordinates $\left.T=T\left(x^{\prime}, y^{\prime}, z^{\prime}, t\right), T x^{\prime}, y^{\prime}, z^{\prime}, t\right)=T^{*}$.

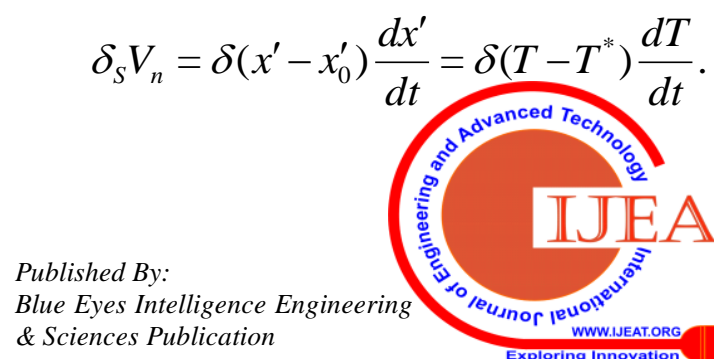


Substituting ( ref stef.10) into ( ref stef.09) gives the desired equation

$$
\begin{aligned}
& {\left[c \rho+L \delta\left(T-T^{*}\right)\right]\left(\frac{\partial T}{\partial t}+\operatorname{vgrad} T\right)} \\
& =\operatorname{div}(\operatorname{kgrad} T)+f,(x, y, z) \in Q
\end{aligned}
$$

Considering the phase transition heat is equivalent to setting the effective heat capacity:

$$
\begin{gathered}
c_{e f}=c+\frac{1}{\rho} L \delta\left(T-T^{*}\right) . \\
\rho c_{e f}\left(\frac{\partial T}{\partial t}+\operatorname{vgrad} T\right)=\operatorname{div}(\operatorname{kgrad} T)+q_{V},
\end{gathered}
$$

$q_{V}$ is the bulk density of internal sources.

In [18] proposes an approach based on the implementation of the internal energy density function $\varepsilon(T)$. The disadvantage of this approach is the lack of a convective component in the proposed thermal conduction equation (see (43), which must be present in the equation for the liquid phase of the polymer state. The disadvantage of this approach is the lack of a convective component in the proposed thermal conductivity equation (see (43), which must be present in the equation for the liquid phase of the polymer state.

We formulate the problem of polymer melt motion in the boundary region of the auger - auger housing, taking into account the convective component. To the equation (43) must be added the equation of motion of the melt film. Since the melt film is thin, it is sufficient to consider the velocity distribution of the fluid along the axial coordinate $Z$. Then we will have:

$$
\begin{aligned}
& \rho\left(\frac{\partial v}{\partial t}+v \frac{\partial v}{\partial z}\right)=-\frac{\partial p}{\partial z}+\mu \frac{\partial^{2} v}{\partial z^{2}} \\
& c_{e f}=c+\frac{1}{\rho} L \delta\left(T-T^{*}\right) . \\
& {\left[\rho c 0_{V}+L\left(T-T^{*}\right)\right]\left(\frac{\partial T}{\partial t}+v \operatorname{grad} T\right)} \\
& =\operatorname{div}(\operatorname{kgrad} T)+q_{V} .
\end{aligned}
$$

Initial conditions:

$$
v(z, 0)=V_{z}(z), T(z, 0)=T_{p}(z, 0) .
$$

Boundary conditions:

$$
\begin{aligned}
& v(t)_{z=z 1}=V_{z}(t), T(t)_{z=z 1}=T_{p}(t), \\
& T\left(r=r_{3}, z, t\right)=T_{\mathrm{ml}, z, t} .
\end{aligned}
$$

The iterative procedure for solving the given system of equations is as follows.

1. First, we obtain the solution of the linearized equation corresponding to

$$
\rho \frac{\partial v^{(0)}}{\partial t}=+\mu \frac{\partial^{2} v^{(0)}}{\partial z^{2}} .
$$

2. The solution obtained for the velocity $v^{(0)}(z, t)$ to solve the equation of the linear part of the equation with respect to temperature

$$
\left[\rho c 0_{V}-L T^{*}\right]\left(\frac{\partial T^{(0)}}{\partial t}+v^{(0)} \operatorname{grad} T^{(0)}\right)
$$

$$
=\operatorname{div}\left(\operatorname{kgrad} T^{(0)}\right)+q_{V} .
$$

3. Find the solution of the equation taking into account the dependence of the heat content coefficient on the melt temperature $c 0_{V}+L\left(T-T^{*}\right)-T^{(1)}(z, t)$.

4. Determine the expression for pressure using $p=\rho R T^{(1)}(z, t)$.

5 . We obtain the solution of the equation (44) in the first approximation, that is, we solve the equation

$$
\begin{aligned}
& \rho \frac{\partial v^{(1)}}{\partial t}=-\frac{\partial p}{\partial z}+\mu \frac{\partial^{2} v}{\partial z^{2}}-N_{v}\left(v^{(0)}, T^{(1)}\right) \\
& N_{v}\left(v^{(0)}, T^{(1)}\right)=v^{(0)}(z, t) \frac{\partial v^{(0)}}{\partial z}+R \rho \frac{\partial T^{(1)}}{\partial z} .
\end{aligned}
$$

6. Repeat steps 2--5 until ||$T^{(m+1)}(z, t)-T^{(m)}(z, t) \|>\Delta$ with the indexes 0 and 1 replaced by $m$ and $m+1$.

Since the iterative procedure for solving nonlinear equations is outlined in the previous sections, we do not give it in this section.

\section{III.RESULTS AND DISCUSSION}

Mathematical modeling of the processes of heating of the screw housing and the polymer mixture in the loading zone and the delay zone is performed.

The solutions of the nonlinear boundary value problems of heat transfer in the loading zone and the delay are obtained, taking into account the nonlinear properties of the coefficient of heat capacity and convective transfer of speed in the delay zone. An algorithm for solving the problem of moving boundaries of the solid and liquid phases of a polymer is proposed.

\section{IV.CONCUSION}

The problem solved,in our opinion, is essential for solving the problem of optimal heating of the polymer mixture in terms of providing high-quality insulating coating of the cable to ultra-high voltages.

\section{REFERENCES}

1. Kim VS The theory and practice of polymer extrusion. M .: Chemistry, Colossus C, 2005. 568 p.

2. Manat BT, Shevtsov PN Development of mathematical model and computer simulation of extrusion process in dosing zone // Theor. and Applied Science. - 2014. - № 5 (13). - P. 9-23.

3. E.V, Subbotin, R.R. Zinnatullin. Experimental determination of rheological characteristics of polymer melt in numerical simulation of extrusion process // Scientific-Techn. newsletter of the Volga region. 2015. - № 4. - P. 28-30.

4. N.M. Trufanova. Melting of polymers in extruders - M .; Izhevsk: Regular and chaotic dynamics, 2009. - 336 p.

5. N.M. Trufanova. Processing of polymers. - Perm: Publisher Perm. state. tech. Univ., 2009. - 158 p.

6. V.N. Mitroshin Structural modeling of the temperature field of a polymer melt in the dosing zone of a single-worm extruder. state. tech. Universities. Ser .: Technical Sciences. - 2006. - № 41. - P. 191-194.

7. E. Ya. Rapoport Optimization of the processes of induction heating of metal. - M .: Metallurgy, 1993. - 279 p.

8. K. Rauvendal, Extrusion of polymers [Text] / K. Rauvendal; ed. AND I. Malkina; trans. with English. - St. Petersburg: Profession, 2008. 768 p. 
9. T.B. Chistyakova , V.Yu. Plonsky , A.N. Pologin. Numerical modeling of process dynamics extrusion of polymeric materials // Mathematical methods in engineering and technology. MMTT-12: Collection of Works 12- her International. scientific Conf. Novgorod, 1999. T. 4. P. 124-125.

10. Automated design and calculation of augermachines: Monograph / M.V. Sokolov, A.S. Klinkov, O.V. Efremov. M .: "Publishing house Machine-building", 2004. 248 p.

11. V.V. Skachkov, R.V. Thorner, J.V. Stungur, S.V. Reutov. Modeling and optimization of polymer extrusion. L .: Chemistry, 1984. 152 p.

12. V.P. Pervadchuk. Processes of motion, heat exchange and phase transformations of non-Newtonian materials in auger-outgoing devices: Dis. ... Dr. Tech. Sciences in special. 05.17.08. Perm, 1984. 377 p.

13. V.M. Bolhovitin. Mathematical modeling of temperature regimes of polymer coating of cable products / V.M. Bolhovitin, K.H. Zelensky // Bulletin of the University of Ukraine, series: Informatics, Computer Science and Cybernetics, №1 (18), 2017 .-- p.50--55.

14. P.M. Talanchuk Simulation of the insulation coating process / PM Talanchuk, V.M. Bolhovitin, K.H. Zelensky // Bulletin of the University of Ukraine, series: Informatics, Computer Engineering and Cybernetics, №1 (18), 2016 .-- p.5--14.

15. K.Ch. Zelensky . Computer modeling of air mixtures in cyclone chambers. K. Ch. Zelensky, V.M. Ignatenko, KS Bovsunovska // Resp. inter. Sat. ASAU, No.1 (22), 2013, pp. 120-134.

16. N.K. Zelenskaya Approximation of cylindrical functions by fractionalrational expressions / N.K. Zelenskaya, K.Ch. Zelensky // Bulletin of the University of Ukraine, series: informatics, computer engineering and cybernetics, №1, 2016. - P.

17. A.A.Samarsky, P.N. Vaibishevich. Computational Heat Transfer, Moscow: URSS Editorial, 2003, 784 p.

18. M.P. Galanin. Solution of three-dimensional non-stationary hea conduction equation by MFE with consideration of phase transitions / M.P. Galanin, A.S. Rodin . // IPM Preprint M.V. Keldysh. 2016. №66.

\section{AUTHORS PROFILE}

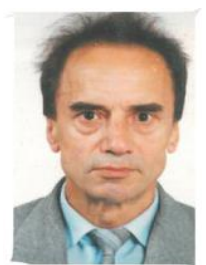

K. Zelensky, Graduated from Kiev University. Taras Shevchenko, Faculty of Mechanics and Mathematics,. Was qualified as mathematician, Candidate of Technical Sciences with a thesis on sympifing of models for complex nonlinear odjects, majoring in Theory of Automatic Control. Associate Professor of Department of Biomedical Cybernetics, National Polytechnic University of Ukraine "Igor Sikorsky Kyiv Polytechnic Institute". Sphere of scientific interests - mathematical modeling and control of complex technological systems, specialist in numerical-analytical methods for solving nonlinear differential equations. Author of more than 100 scientific publications, 15 textbooks, 2 monographs (co-authored). Monograph "Methods for the Study of Electrotechnical Systems and Complexes", 2019, 298 p.

Under the guidance of C. Zelensky are defended 4 and 3 dissertations are being carried out for the degree of Candidate of Technical Sciences in the specialty "Computer Sciences".

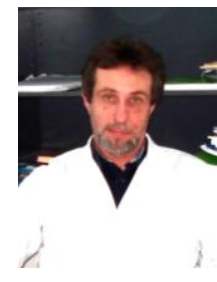

Evgen Nastenko, Doctor of Biological Sciences, Senior Researcher, Head of the Department of Biomedical Cybernetics, Faculty of Biomedical Engineering, National Polytechnic University of Ukraine "Igor Sikorsky Kyiv Polytechnic Institute". Member of the Specialized Scientific Council 14.01.04 - Cardiovascular Surgery of the State Institution "National M. Amosov' Institute of Cardiovascular Surgery". Member of the Specialized Scientific Council 03.00.20 - Biotechnology, Faculty of Biotechnology and Biotechnics, Igor Sikorsky Kyiv Polytechnics Institute. Head of scientific projects: 1. Development of a robust method for automatic identification of anatomical features of the heart for cardiac analysis. Samsung Electronics Ukraine Company. Contract dated 26.06.2014.r. 2. Blood pressure assessment. Samsung Electronics Ukraine Company. Contract dated 09.10.2018 .3. "Methods and Models for the Identification of the State of Biological Objects" (2017-2022He has 306 publications, of which 284 are scientific, 3 chapters in three monographs, 13 patents, including 156 scientific papers published in national and international peer-reviewed professional journals, 17 articles in Scopus. Under the guidance of Dr. E.A. Nastenko are defended 6 and 2 dissertations are being carried out for the degree of Candidate of Technical Sciences in the specialty "Computer Sciences".

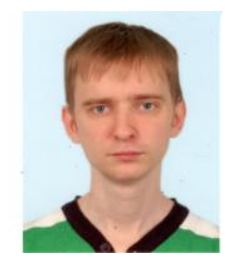

V. Bolhovitin, Vasily Bolhovitin graduated from the Open International University of Human Development "Ukraine" with a degree in computer systems and networks and received a master's degree in computer systems analytics. Graduate student in the department of computer systems and networks. Administrator of the computer center of the Institute of Computer Technology of the University of Ukraine. Lecturer, Department of Computer Systems and Networks, Institute of Computer Technology. His research interests include computer modeling of processes described by nonlinear heat and mass transfer equations (Navies - Stokes equations), computer modeling of intelligent systems (neural networks), and the creation of intelligent knowledge bases. He has three publications in scientific journals. Article "Mathematical modeling of the process of cooling of polymer melts", Bulletin of the University of Ukraine, 2018, No. 20. P. 34-48.

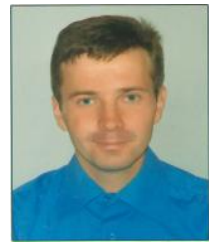

A. Pavlov., Pavlov Alexander was qualified as an engineer at the Kyiv Polytechnic Institute (KPI), specializing in Intelligent Decision-Making Systems (2002). In 2007 he got $\mathrm{PhD}$ with a thesis on selforganizing algorithms in the tasks of the increasing information value for geometrical models. Currently is an senior lecturer of computer science at department of descriptive geometry, engineering and computer graphics, National Technical University of Ukraine «Igor Sikorsky Kyiv Polytechnic Institute», the physics and mathematics faculty. His research interests are in the field of complicated systems modeling, causal analysis of interconnected processes, pattern recognition, decision support methods in medical diagnostic tasks, methods for optimizing personalized strategies 